\title{
Pollution and Life Expectancy: How environmental policy can promote growth
}

\author{
Xavier Pautrel ${ }^{*}$ \\ 2008, April 17
}

\begin{abstract}
This article investigates the influence of environmental policy $(E P)$ on growth in an $A K$-type growth model, when finite lifetime is introduced and the link between pollution and life expectancy (through the detrimental impact of pollution on health) is taken into account.

Using an overlapping generations model à la Blanchard (1985), we demonstrate that finite lifetime introduces a "generational turnover effect" which modifies the influence of the EPon growth. Thus, when lifetime is finite and independent from pollution, we show that the "generational turnover effect" limits the detrimental impact of the EP on growth, if agents smooth their consumption over time. When pollution negatively influences life expectancy through health, we demonstrate that the "generational turnover effect" is magnified and that the relation between the EP and growth is reversed U-shaped in the steady-state. In this case, we show that the environmental policy is more likely to promote growth (i.e. it stimulates growth for a wider range of environmental taxes) when the impact of pollution on health is important and/or public expenditures in health is low. Finally, using numerical simulations, we find that for the value of parameters chosen the $E P$ will be more likely to promote growth when agents smooth consumption over time.
\end{abstract}

Keywords : Growth; Environment; Overlapping generations

\footnotetext{
*Nantes Atlantique Université, Laboratoire d'Économie et de Management de Nantes (LEM), Institut d'Économie et de Management de Nantes - IAE, Chemin de la Censive du Tertre, BP 81307, 44313 Nantes Cedex 3, France. Mail: xavier.pautreleuniv-nantes.fr

${ }^{\dagger}$ An earlier version appeared in 2007 as working paper of the Fondazione ENI E. Mattei n.155. I thank anonymous referees for their remarks on an earlier version of this paper. The usual disclaimer applies.
} 


\section{INTRODUCTION}

Even if the link between environmental policy and growth is a longstanding debate, recent reports upon climate change and the very quick and unbridled industrialization of the biggest economies in the world, such as China and India, ${ }^{1}$ have dramatically emphasized the pressing necessity to implement efficient and global environmental policy with an eye towards economic performances.

This article investigates the effect of environmental policy on growth in an $A K$-type growth model introducing finite lifetime and the link between pollution and life expectancy (through the detrimental impact of pollution on health). It gets away from two observations about the existing literatures on the environment and growth. First, as highlighted by Ricci (2007) in a recent survey, a better environmental quality leads to a lower growth rate in both basic $A K$ and $\mathrm{R} \& \mathrm{D}$-driven growth models: reducing pollution to increase the environmental quality turns away resources to investment and therefore drags down growth. To offset this crowding-out effect, it is necessary, for example, either to take into account the external influences of the environment on productivity or some policy-induced adjustments (see Ricci, 2007, p.694), either to assume elastic labor supply (see Hettich, 1998) or constant returns to scale in the pollution abatement sector (see Michel and Rotillon, 1995). One of the contribution of our article is to demonstrate that the turnover of generations (births and deaths) is sufficient to limit and even to offset the negative effect of the environmental policy in the $A K$ framework.

Second observation which motivates the paper: the detrimental influence of pollution on health and life expectancy is one of the most well-documented phenomenon in the field and one of the most striking features of the negative impact of pollution on individuals. In its 2002 report, the World Health Organization estimates that about 800,000 deaths and 7.9 million lost life-years worldwide are attributable to air pollution. This adverse effect is

\footnotetext{
${ }^{1}$ From the World Development Indicators (the World Bank), in 2005, India and China grew respectively at an annual rate of $9.23 \%$ and $10.20 \%$ (in stable increase) while the World and the High income countries grew respectively at $3.48 \%$ and $2.66 \%$.
} 
especially true of particulate matter (PM), particles small enough to be inhaled in the lung, which causes " $5 \%$ of trachea, bronchus and lung cancer, $2 \%$ of cardio-respiratory mortality and $1 \%$ of respiratory infections mortality globally." (WHO 2002, p. 69). Kampa and Castanas (2007), reviewing the detrimental effects of a number of air pollutants in human health (on respiratory, cardiovascular, nervous, urinary and/or digestive system), find that short- and long-term exposures are linked with premature mortality and reduced life expectancy. Neuberg et al. (2007) report for Vienna, during the period 2000-2004, the lethal effect of the smallest particulate matters $\mathrm{PM}_{2.5}$ (particulate matter smaller than 2.5 microns) and of $\mathrm{NO}_{2}$ even at relatively low levels of these pollutants: the total mortality increase for $10 \mu \mathrm{gm}^{-3}$ of $\mathrm{PM}_{2.5}$ and $\mathrm{NO}_{2}$ is equal respectively to $2.6 \%$ and $2.9 \%$, mainly because of cardiopulmonary and cerebrovascular causes. They conclude that the objectives fixed in terms of $\mathrm{PM}_{2.5}$ and $\mathrm{NO}_{2}$ by the European Union in 2010 will be unable to prevent serious health effects. $^{2}$ For water pollution, Valent et al. (2004) find that inadequate water and sanitation are responsible for $5.3 \%$ of deaths and $3.5 \%$ of disability-adjusted life years (DALYs) for young people aged 0-14 years in Europe. ${ }^{3}$ Furthermore, as demonstrated by Bloom et al. (2004), Bloom and Canning (2005), or studies in López-Casanovas et al. (2005), health is a key factor of economic growth: it increases productive efficiency, it extends life expectancy, it promotes learning capacity and therefore fosters knowledge accumulation (Howitt, 2005). Consequently, health must be taken into account to study the link between the environment and growth. However, few environment-growth analysis integrate it explicitly. A notable exception is the contribution by Gradus and Smulders (1993). In a section of their article, using a model of endogenous growth à la Lucas (1988), they justify the negative impact of

\footnotetext{
${ }^{2}$ For more references on empirical studies demonstrating that air pollution shortens life expectancy, see the surveys by Brunekreef and Holgate (2002) and by Englert (2004). See also references in Gutierrez (2005).

${ }^{3}$ Several other studies link the presence of some pollutants in drinking water to the increase in cancer risk: tetrachloroethylene (PCE) and lung cancer in Massachusetts (Paulu et al., 1999), arsenic and bladder cancer in Finland (Kurttio et al., 1999) and the use of chlorine for water treatment and bladder and rectal cancer in the United States (Morris, 1995). Industrial or chemical pollution is also reported as increasing the cases of cancer for adults and children contributing to shorter life expectancy (Nadal et al., 2004; Chen and Liao, 2005; Schuhmacher and Domingo, 2006).
} 
pollution on human capital accumulation by its effect on health that limits the ability of agents to educate. Despite the interest of their analysis, two limitations may be emphasized which call for further investigations. First, they broadly introduce pollution in the education sector (the core of growth) as a simple component of the human capital depreciation. Microfoundations would be necessary to make the underlying mechanisms clearer. Furthermore, they base their analysis on the link between health and education. Nevertheless, while this link sounds logical and the influence of the education on health has been empirically established (see Grossman and Kaestner, 1997), there is a lack of empirical evidence on the causality between health and education (see Ding et al., 2005). Second, they wrote about the $A K$ model that "crowding-out effects [due to the environmental policy] may be compensated by other effects as soon as possibilities for substitution and variable capital productivity are taken into account." (Gradus and Smulders, 1993, p.36). They continue writing that a "key assumption of the Rebelo model is the absence of factor substitution [... and that ...] productivity effects are more likely to apply to labor than to capital." (Gradus and Smulders, 1993, p.36). Consequently, they conclude that the $A K$ model does not enable to have such a compensated effect and they use the Lucas (1988) endogenous growth model. One contribution of our article, with respect to their work, is to highlight a mechanism which compensates the crowding-out effect of the $E P$ in the $A K$ model while neither substitution between polluting and non-polluting factors, nor variable capital productivity are assumed.

Consequently, in this article, we re-examine the relation between the environment and growth, taking into account finite lifetime and the impact of pollution on life expectancy, without making any assumption about a positive effect of the environmental quality on factors productivity nor assuming factor substitution. For this purpose, we use an overlapping generations model à la Blanchard (1985) in which we model explicitly the link between pollution and the lifetime of the agents. Long-run growth is driven by externalities from the aggregate stock of physical capital ( $A K$ model à la Romer, 1986) and the life expectancy of 
agents depends on public health which is influenced negatively by the level of pollution and positively by public health expenditures.

In this $A K$ model with environmental and health concerns, we first demonstrate that finite lifetime introduces a "generational turnover effect" ${ }^{4}$ which modifies the influence of the environmental policy on growth, even if pollution does not influence life expectancy. This "generational turnover effect" appears because at each date a new generation is born and a cross-section of the existing population dies. Because new agents born without financial assets, their consumption is lower than the average consumption and therefore the "generational turnover effect" reduces the growth rate of the aggregate consumption. When lifetime is finite and is not influenced by pollution, this "generational turnover effect" limits the detrimental impact of the environmental policy $(E P)$ on growth, if agents smooth their consumption over time. Indeed, by increasing the tax rate, the EP depresses the interest rate and therefore the accumulation of physical capital and the growth rate of the aggregate consumption. Nevertheless, to smooth their consumption over time and to compensate the decrease in the interest rate, agents reduce their propensity to consume out of wealth and save more today. Investment increases and this positive effect limits the direct negative impact of the tax on the interest rate. Conversely, when agents do not smooth their consumption over time, they increase their propensity to consume out of wealth to compensate their loss of consumption today. Their saving decreases and the investment is reduced: the detrimental impact of the environmental tax on physical capital accumulation is reinforced.

We also demonstrate that, when the detrimental effect of pollution on health and life expectancy is taken into account, the "generational turnover effect" impacts the outcome of the EP through a second channel: life expectancy. In this case, whatever the intertemporal elasticity of substitution of consumption, the relation between the environmental policy and growth becomes reversed U-shaped in the steady-state. For low levels of taxation, the environmental policy promotes growth because the positive influence of a lower net flow

\footnotetext{
${ }^{4}$ The term comes from Heijdra and Ligthart (2000).
} 
of pollution on health and life expectancy leads the generational turnover effect to offset the crowding-out effect on the investment due to the increasing tax. For higher levels, the environmental policy becomes harmful to growth because the positive effect on health is defeated by the crowding-out effect. ${ }^{5}$ We show that the lower the public expenditures in health (related to GDP) and/or the higher the impact of pollution on health, the more the environmental policy is likely to promote growth, because the greater the room for health improvements due to the EP. Finally, we make numerical simulations to investigate the influence of the intertemporal rate of substitution of consumption on our result. We find that, for the value of parameters chosen, the environmental policy is more likely to promote growth when agents smooth consumption over time.

The article is structured as follows. Section 2 gives the basic framework of our model and section 3 formalizes the link between pollution, health and life expectancy. Section 4 investigates the steady-state equilibrium of the economy. Section 5 looks into the impact of environmental taxation on growth and section 6 goes into detail using numerical simulations. Section 7 draws this article to a conclusion.

\section{THE ECONOMY'S STRUCTURE}

Let us consider an overlapping generations model à la Blanchard (1985) with human capital accumulation and environmental concerns. Time is continuous. Each individual born at time $s$ faces a constant probability of death per unit of time $\lambda_{s} \geq 0$. Consequently, his life expectancy is $1 / \lambda_{s}$. When $\lambda_{s}$ increases, the horizon of the economy becomes shorter. At time $s$, a cohort of size $\lambda_{s}$ is born. This cohort has a size equal to $\lambda_{s} e^{-\lambda_{s}(t-s)}$ at time $t$. The constant population is equal to $L_{t} \equiv \int_{-\infty}^{t} \lambda_{s} e^{-\lambda_{s}(t-s)} d s$ at time $t$. For convenience it is normalized to

\footnotetext{
${ }^{5}$ Using a discrete-time overlapping generations model, Ono (2003) also demonstrates that two opposite forces link environmental policy and growth, because a higher quality of environment is bequeathed to future generations. Nevertheless, he emphasizes that his result is very specific and not always occurs even if environmental quality is bequeathed to future generations because it " depends heavily on [...] the distinction between the short-lived and the long-lived governments, and [...] the specific form of the utility function" (Ono, 2003, p.214). He demonstrates that, when the government spends environmental tax revenue to finance environmental investment, the growth rate is always decreasing in environmental tax.
} 
unity. There is no bequest motive and there are insurance companies. ${ }^{6}$

Contrary to Blanchard (1985), we assume that the probability of death for an agent born at time $s$ depends negatively on the public health in the economy when he is born $\varepsilon_{s} \cdot{ }^{7}$ To simplify we pose $\lambda_{s}=\varepsilon_{s}^{-1}$.

The expected utility function of an agent born at $s \leq t$ is:

$$
\int_{s}^{\infty} U\left(c_{s, t}, \mathcal{P}_{t}\right) e^{-\left(\rho+\lambda_{s}\right)(t-s)} d t
$$

where $c_{s, t}$ denotes consumption in period $t$ of an agent born at time $s, \mathcal{P}_{t}$ is the net pollution flow at time $t, \rho \geq 0$ is the rate of time preference and

$$
U\left(c_{s, t}, \mathcal{P}_{t}\right)= \begin{cases}\frac{\left[c_{s, t} \mathcal{P}_{t}^{-\phi}\right]^{1-1 / \sigma}-1}{1-1 / \sigma} & \sigma \neq 1, \\ \ln c_{s, t}-\phi \ln \mathcal{P}_{t} & \sigma=1,\end{cases}
$$

with $\phi$ measures the weight in utility attached to the environment, that is environmental care. $\sigma$ is the elasticity of intertemporal substitution.

Due to the simple demographic structure, all individual variables are additive across individuals. Consequently, the aggregate consumption equals

$$
C_{t}=\int_{-\infty}^{t} c_{s, t} \lambda_{s} e^{-\lambda_{s}(t-s)} d s,
$$

The aggregate production function is defined by:

$$
Y_{t}=\tilde{A}_{t} K_{t}^{\alpha} L_{t}^{1-\alpha}, \quad 0<\alpha<1
$$

with $Y_{t}$ being the aggregate final output. $K_{t}$ is the aggregate stock of physical capital and $L_{t}$ is the amount of labor (equal to the population). As discussed in Romer (1986), we assume

\footnotetext{
${ }^{6}$ The first assumption is made because here death may be interpreted as the termination of a family dynasty and therefore adults who die do not care about what occurs beyond their death. The second assumption is made to avoid unintented bequests.

${ }^{7}$ As emphasized by a reviewer, empirical evidence suggests that life expectancy should depend on the current date rather than the birth date. The assumption is made here for convenience in order to not complicate the exposition of the model. Results are not modified because as we will demonstrate in section 4, the probability of death is constant over time and similar for all agents.
} 
that there exists external effects of aggregate capital on productivity: $\tilde{A}_{t}=A K_{t}^{1-\alpha}$, where $A>0$ is a constant parameter. Consequently, the aggregate production function reduces to:

$$
Y_{t}=A K_{t} L_{t}^{1-\alpha}
$$

Finally we assume that the government implements two types of policy: a health policy which consists in publicly providing health services to agents and an environmental policy which consists in taxing the flow of pollution from firms. The government is assumed to balance its budget constraint all the time (see below).

\section{Pollution, Health ANd Life expectancy}

Following Gradus and Smulders (1993), pollution flow is assumed to increase with the stock of physical capital $K$ and reduces with abatement activities $D$ :

$$
\mathcal{P}_{t}=\left[\frac{K_{t}}{D_{t}}\right]^{\gamma}, \quad \gamma>0
$$

We consider that public health at time $s$ is influenced negatively by the net flow of pollution and positively by the part of public health expenditures in GDP: ${ }^{8}$

$$
\varepsilon_{s}=\frac{\beta \theta}{\delta \mathcal{P}_{s}^{\psi}}
$$

where $\theta$ is the exogenous part of the aggregate final output that the government uses to publicly provide public-health services. $\beta>0$ is the productivity of the health sector, $\delta$ is a positive parameter. $\psi>0$ measures the influence of pollution on public health: a higher value of $\psi$ means that pollution harms more public health. This assumption imposes that $\mathcal{P}>1$.

Abatement activities use final output (one for one) so the final market clearing condition is:

$$
(1-\theta) Y_{t}=C_{t}+\dot{K}_{t}+D_{t}
$$

\footnotetext{
${ }^{8}$ We follow empirical studies which use in their estimations expenditures in health as a percentage of GDP rather than the amount of expenditures in health (see Currais and Rivera (1999), Currais and Rivera (2003) for example).
} 


\section{The General EQUilibrium AND the BALANCED GROWTH PATH}

In this section, we derive the dynamical system which summarizes the intertemporal evolution of the economy and the steady-state defined as a balanced-growth path equilibrium where $C, Y, D$ and $K$ evolve at a common positive rate of growth.

As previously noted, besides its health policy, the government also implements an environmental policy which consists of taxing the net flow of pollution by firms and transferring to them the fruit of the taxes to fund their abatement activities. Consequently, firms under perfect competition pay a pollution tax on their net pollution $\mathcal{P}_{t}$ and they choose their abatement activities $D_{t}$ (whose cost equals $D_{t}$ ) and the amount of factors which maximize their profits $\pi_{t}=Y_{t}-r_{t} K_{t}-w_{t} L_{t}-\vartheta_{t} \mathcal{P}_{t}-D_{t}+T_{t}^{p}$ where $\vartheta_{t}$ is the pollution tax rate and $T_{t}^{p}$ denotes transfers from the public sector with $T_{t}^{p}=\vartheta_{t} \mathcal{P}_{t}$. Firms take as given these transfers and pay each production factor at its marginal productivity to maximize profit:

$$
\begin{aligned}
& r_{t}=\alpha A L_{t}^{1-\alpha}-\vartheta_{t} \gamma \frac{\mathcal{P}_{t}}{K_{t}} \\
& w_{t}=(1-\alpha) A K_{t} L_{t}^{-\alpha}
\end{aligned}
$$

and

$$
D_{t}=\vartheta_{t} \gamma \mathcal{P}_{t}
$$

From (3) and (7), we obtain $\mathcal{P}_{t}=\left[\gamma \frac{\vartheta_{t}}{K_{t}}\right]^{-\gamma /(1+\gamma)}$. Because in the steady-state, the quality of the environment must be constant, $\vartheta_{t}$ must evolve as the physical capital. Intuitively, it increases over time to encourage firms to increase abatement activities to limit pollution which rises with the physical capital stock. Consequently, we define $\tau \equiv \vartheta_{t} / K_{t}$, the environmental tax normalized by the physical capital, and following Oueslati (2002) we assume that it is fixed by the government and has no transitional dynamics. ${ }^{9}$ Consequently, we obtain:

$$
\mathcal{P}=\Phi(\tau)^{-\gamma}
$$

\footnotetext{
${ }^{9}$ Here this assumption is of no consequence inasmuch as the $A K$ model has no transitional dynamics. See below Figure 1.
} 
with $\Phi(\tau) \equiv[\gamma \tau]^{\frac{1}{1+\gamma}}$ is an increasing function of $\tau$. The net flow of pollution $\mathcal{P}$ is constant over time. Then, equations (6) and (7) may be re-written as (remembering that population is normalized to unity):

$$
\begin{aligned}
& r=\alpha A-\Phi(\tau) \\
& w_{t}=(1-\alpha) A K_{t}
\end{aligned}
$$

and

$$
D_{t}=\Phi(\tau) K_{t}
$$

Because we imposed $\mathcal{P}>1$ in the previous section, equation (3) implies $D_{t}<K_{t}$. Consequently, from (10) we have $\Phi(\tau) \in] 0,1[$. Furthermore, $r>0$ imposes $\alpha A>\Phi(\tau)$.

From (4) and because we assumed $\lambda_{t}=\varepsilon_{t}^{-1}$, the probability of death is independent of time and defined by:

$$
\lambda=\frac{\delta \Phi(\tau)^{-\psi \gamma}}{\beta \theta} \equiv \mathcal{L}(\tau)
$$

where $\mathcal{L}(\tau)$ is a decreasing function of $\tau$.

Households face the following budget constraint:

$$
\dot{a}_{s, t}=[r+\lambda] a_{s, t}+w_{t}-c_{s, t}
$$

where $a_{s, t}$ is the financial wealth in period $t$ and $\omega_{t}$ represents the wage rate per effective unit of labor. $r+\lambda$ represents the interest rate $r$ plus the amount received from the insurance company when the agent survives, which is equal to the probability of death $\lambda$. In addition to the budget constraint, there exists a transversality condition which must be satisfied to prevent households from accumulating debt indefinitely:

$$
\lim _{v \rightarrow \infty}\left[a_{s, v} e^{-(r+\lambda)(v-t)}\right]=0
$$


The representative agent chooses the time path for $c_{s, t}$ by maximizing (1) subject to (12). It yields

$$
\dot{c}_{s, t}=\sigma[r-\rho] c_{s, t}
$$

Integrating (12) and (14) and combining the results gives the consumption at time $t$ of an agent born at time $s$ :

$$
c_{s, t}=\Delta(\tau)\left[a_{s, t}+\omega_{s, t}\right]
$$

where $\omega_{s, t} \equiv \int_{t}^{\infty} w_{\nu} e^{-(\nu-t)(r+\lambda)} d \nu$ is the present value of lifetime earning and

$$
\Delta(\tau)=(1-\sigma) \alpha A-(1-\sigma) \Phi(\tau)+\sigma \rho+\mathcal{L}(\tau)
$$

is the propensity to consume the overall individual revenue and is constant over time. ${ }^{10}$ By definition the propensity to consume must be positive, consequently we consider that the probability of death is high enough to ensure that $\Delta(\tau)$ is always positive. ${ }^{11}$ It implies:

$$
\mathcal{L}(\tau)>(1-\sigma)[\Phi(\tau)-\alpha A]-\sigma \rho
$$

Because the interest rate must be positive (that is $\alpha A>\Phi(\tau)$ from equation 8 ) this conditions is always verified whatever $\mathcal{L}(\tau)$ when $\sigma \leq 1$.

The aggregate consumption equals

$$
C_{t}=\int_{-\infty}^{t} c_{s, t} \lambda e^{-\lambda(t-s)} d s=\Delta(\tau)\left[K_{t}+\Omega_{t}\right]
$$

with $\Omega_{t} \equiv \int_{-\infty}^{t} \omega_{s, t} \lambda e^{-\lambda(t-s)} d s$, and the aggregate stock of physical capital is defined by

$$
K_{t}=\int_{-\infty}^{t} a_{s, t} \lambda e^{-\lambda(t-s)} d s
$$

\footnotetext{
${ }^{10}$ When the interest rate is not constant over time, we have $\left[\int_{t}^{\infty} e^{-(\sigma \rho+\lambda)(\nu-t)-(1-\sigma) \int_{t}^{\nu} r_{\mu} d \mu} d \nu\right]^{-1}$ which is not constant.

${ }^{11}$ This guarantees, introducing equation (14) in the expression of the intertemporal utility (equation 1), that this utility, expressed as $\frac{1}{1-1 / \sigma} \int_{s}^{\infty}\left\{\mathcal{P}_{t}^{-\phi(1-1 / \sigma)} c_{s, s}^{1-1 / \sigma} e^{(\sigma-1)(r-\rho)(t-s)}-1\right\} e^{-\left(\rho+\lambda_{s}\right)(t-s)} d t$, is finite.
} 
Differentiating (16) with respect to time and using the expression of $d K_{t} / d t$ and $d \Omega_{t} / d t$ with equations (8) and (11) gives the law of motion of the aggregate consumption:

$$
g_{C, t} \equiv \dot{C}_{t} / C_{t}=\sigma[\alpha A-\Phi(\tau)-\rho]-\mathcal{L}(\tau) \Delta(\tau) K_{t} / C_{t}
$$

Aggregate consumption growth differs from individual consumption growth (equation 14) by the term $\mathcal{L}(\tau) \Delta(\tau) K_{t} / C_{t}$ which represents what Heijdra and Ligthart (2000) called the "generational turnover effect". This effect appears because at each date a new generation is born and a cross-section of the existing population dies. Because new agents born without financial assets, their consumption is lower than the average consumption and therefore the "generational turnover effect" reduces the growth rate of the aggregate consumption. This generational effect rises with the probability of death $\mathcal{L}(\tau)$ : on one hand, agents die at a higher frequency (that increases the generational turnover) and on the other hand the propensity to consume out of wealth $\Delta(\tau)$ increases due to the shorter horizon. The rise of the propensity to consume out of wealth, by itself, reinforces the "generational turnover effect" and augments current aggregate consumption: agents prefer to consume today than tomorrow and therefore saving, investment and growth are lower. These mechanisms underlying the "generational turnover effect" are central to understand the impact of the environmental policy on growth when lifetime is finite, even if lifetime is not affected by pollution. $^{12}$

Furthermore, from (5), (2) and (7) the law of motion of the physical capital is:

$$
g_{K, t} \equiv \dot{K}_{t} / K_{t}=(1-\theta) A-C_{t} / K_{t}-\Phi(\tau)
$$

Consequently, denoting the consumption-capital ratio by $x_{t}$, the economy is summarized

\footnotetext{
${ }^{12}$ It is clear from equation (15) that the environmental taxation influences the propensity to consume out of wealth, independently from the variations of the probability of death $\mathcal{L}(\tau)$, and therefore will modify the effect of the environmental policy on growth even if finite lifetime is exogenous. Cf. infra for a formal proof.
} 
by the two following equations which both depends on $x_{t}$ :

$$
\begin{aligned}
g_{C, t} & \equiv \frac{\dot{C}_{t}}{C_{t}}=\sigma[\alpha A-\Phi(\tau)-\rho]-\mathcal{L}(\tau) \Delta(\tau) x_{t}^{-1} \\
g_{K, t} & \equiv \frac{\dot{K}_{t}}{K_{t}}=(1-\theta) A-x_{t}-\Phi(\tau)
\end{aligned}
$$

The first equation is an increasing function of the ratio aggregate consumption to aggregate capital $x$ and the second one is a decreasing function. When they intersect they define a unique consumption-capital ratio $x^{\star}$ which corresponds to the steady-state equilibrium of the economy, where $C, K, D$ and $Y$ grow at a common positive rate $g^{\star}$ (the star denotes steady-state).

Figure 1: The steady-state

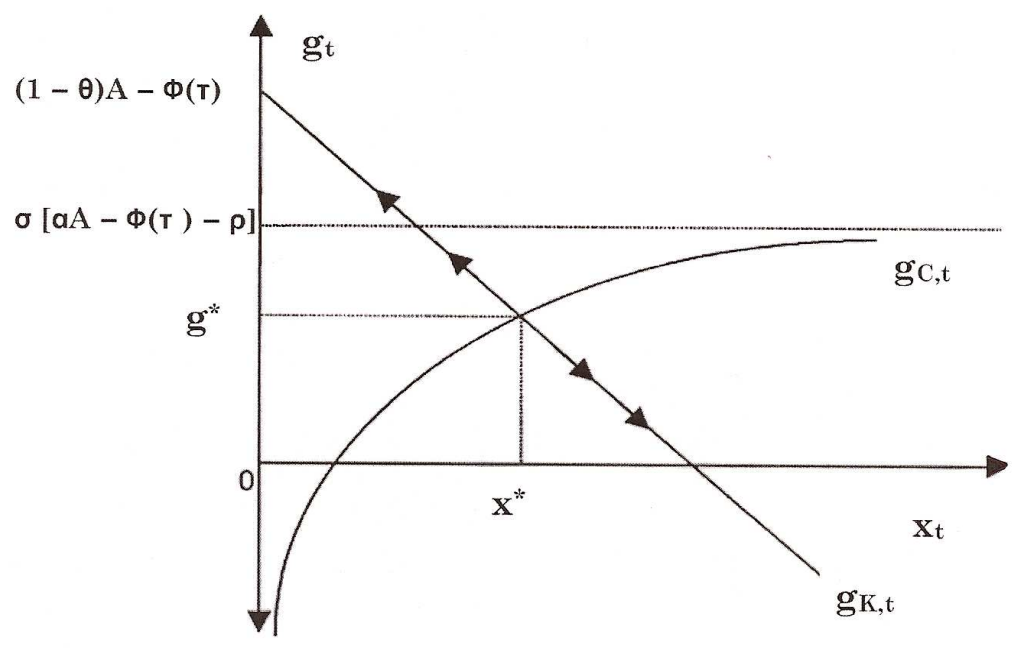

Formally, $x^{\star}$ is the positive solution of the second-order equation $x^{\star 2}-\Omega(\tau) x^{\star}-\mathcal{L}(\tau) \Delta(\tau)=$ 0 that is

$$
x^{\star}=\frac{1}{2}\left\{\Omega(\tau)+\sqrt{\Omega(\tau)^{2}+4 \mathcal{L}(\tau) \Delta(\tau)}\right\}
$$

where $\Omega(\tau) \equiv(1-\theta-\sigma \alpha) A-(1-\sigma) \Phi(\tau)+\sigma \rho>0{ }^{13}$ and $\Delta(\tau) \equiv(1-\sigma)[\alpha A-\Phi(\tau)]+\sigma \rho+\mathcal{L}(\tau)$.

\footnotetext{
${ }^{13}$ We impose that $\Omega(\tau)$ is positive because it is the expression of $x^{\star}$ when lifetime is infinite. Cf infra.
} 
Note that when agents want to smooth their consumption over time $(\sigma<1)$, the ratio aggregate consumption to aggregate capital in the steady-state $\left(x^{\star}\right)$ is negatively influenced by the environmental taxation because a higher tax reduces the rewards to capital and therefore drops the non-human wealth. Agents willing to smooth their consumption over time, they reduce their propensity to consume out of wealth and increase their saving to compensate the reduction of wealth in the future. ${ }^{14}$ This phenomenon promotes saving, investment and therefore the accumulation of physical capital and reduces the aggregate consumption: the ratio aggregate consumption to aggregate capital decreases. Furthermore, due to consumption smoothing, the increase in the tax influences less aggregate consumption growth (through the increase in the interest rate captured by $\sigma \Phi(\tau)$ in the expression of $\Omega(\tau)$ ) than physical capital growth (through the decrease in the part of the aggregate capital stock used in private abatement activities captured by $\Phi(\tau)$ in the expression of $\Omega(\tau))$. The ratio aggregate consumption to agregrate physical capital reduces to equalize the growth rates.

The growth rate in the steady-state is unique and defined as a function of the environmental $\operatorname{tax} \tau$ :

$$
g^{\star}=(1-\theta) A-\Phi(\tau)-\frac{1}{2}\left\{\Omega(\tau)+\sqrt{\Omega(\tau)^{2}+4 \mathcal{L}(\tau) \Delta(\tau)}\right\}
$$

with

$$
\begin{aligned}
& \Phi(\tau) \equiv[\gamma \tau]^{\frac{1}{1+\gamma}} \\
& \mathcal{L}(\tau) \equiv \frac{\delta}{\beta \theta} \Phi(\tau)^{-\psi \gamma} \\
& \Delta(\tau) \equiv(1-\sigma)[\alpha A-\Phi(\tau)]+\sigma \rho+\mathcal{L}(\tau)>0 \\
& \Omega(\tau) \equiv(1-\theta-\sigma \alpha) A-(1-\sigma) \Phi(\tau)+\sigma \rho>0
\end{aligned}
$$

Figure 1 represents the steady-state equilibrium of the economy and shows that there is no transitional dynamics (arrows indicate the evolution of the ratio aggregate consumption to aggregate physical capital if the economy would not be at the steady-state). If the economy starts with a consumption-capital ratio lower than $x^{\star}$, the growth rate of the physical

\footnotetext{
${ }^{14}$ The propensity to consume out of wealth is also reduced by the fact that an increase in the environmental taxation reduces the probability of death $\mathcal{L}(\tau)$, whatever the value of the intertemporal elasticity of substitution of consumption $\sigma$.
} 
capital is higher than the growth rate of consumption and therefore the consumption-capital ratio decreases. This increase in the physical capital rate of growth leads to the violation of the transversality condition (13) at a moment of time. Consequently, the economy can not start at a consumption-capital ratio lower than the steady-state value. Similarly, if the economy starts at a consumption-capital ratio higher than $x^{\star}$, the growth rate of consumption is higher than the growth rate of capital and the consumption-capital ratio increases up to a point where the capital stock attains 0 . To verify the output market equilibrium (5), consumption must jump to 0, violating the Euler equation (17). Consequently, the economy can not start at a consumption-capital ratio higher than the steady-state value. Therefore, there is no transitional dynamics in this economy. ${ }^{15}$

\section{ENVIRONMENTAL TAXATION AND GROWTH}

To investigate the influence of the environmental taxation on growth, we derive (19) with respect to $\tau$. It gives:

$$
\frac{\partial g^{\star}}{\partial \tau}=\frac{\partial g^{\star}}{\partial \Phi(\tau)} \frac{\partial \Phi(\tau)}{\partial \tau}
$$

where

$$
\begin{aligned}
\frac{\partial g^{\star}}{\partial \Phi(\tau)}=\frac{1}{2}\left\{(1-\sigma) \frac{2 \mathcal{L}(\tau)+\Omega(\tau)}{\sqrt{\Omega(\tau)^{2}+4 \Delta(\tau) \mathcal{L}(\tau)}}\right. & \\
& \left.-(1+\sigma)+2 \gamma \psi \frac{\mathcal{L}(\tau)[\Delta(\tau)+\mathcal{L}(\tau)]}{\Phi(\tau) \sqrt{\Omega(\tau)^{2}+4 \Delta(\tau) \mathcal{L}(\tau)}}\right\}
\end{aligned}
$$

and

$$
\begin{aligned}
& \Phi(\tau) \equiv[\gamma \tau]^{\frac{1}{1+\gamma}} \\
& \mathcal{L}(\tau) \equiv \frac{\delta}{\beta \theta} \Phi(\tau)^{-\psi \gamma} \\
& \Delta(\tau) \equiv(1-\sigma)[\alpha A-\Phi(\tau)]+\sigma \rho+\mathcal{L}(\tau)>0 \\
& \Omega(\tau) \equiv(1-\theta-\sigma \alpha) A-(1-\sigma) \Phi(\tau)+\sigma \rho>0
\end{aligned}
$$

\footnotetext{
${ }^{15}$ This explanation follows Kosempel (2004).
} 
The function $\Phi(\tau)$ being an increasing function of $\tau$, the influence of the environmental tax is given by the sign of (20).

To clarify as much as possible the mechanisms which operate when lifetime is taken into account and pollution affects life expectancy (through health), we first expose the case where the lifetime of agents is infinite and the case where lifetime is finite but is not affected by pollution. Hence, we back to the general case exposed in the previous sections and we examinate the effect of environmental taxation on growth when pollution affects health and health influences the lifetime of agents.

\subsection{Lifetime is infinite}

In this case, the probability of death is independent of the environmental policy because it is null: $\mathcal{L}(\tau)=0$. The system (18) becomes:

$$
\begin{aligned}
& g_{C, t}=\sigma[\alpha A-\Phi(\tau)-\rho] \\
& g_{K, t}=(1-\theta) A-x_{t}-\Phi(\tau)
\end{aligned}
$$

The growth rate of the aggregate consumption becomes independent from $x$ and consequently is an horizontal curve which shifts downward when $\tau$ increases. The growth rate of the aggregate capital remains a decreasing curve with respect to $x$ and shifts on the left when $\tau$ increases. The variation of $x^{\star}$ depends on the value of $\sigma$ with respect to 1 because $g_{C}^{\star}=g_{K}^{\star}$ gives $x^{\star}=\Omega(\tau) \equiv(1-\theta-\sigma \alpha) A+(\sigma-1) \Phi(\tau)+\sigma \rho>0$. When $\sigma<1$ (respectively $\sigma>1$ ), $x^{\star}$ decreases (respectively increases) with $\tau$. Nevertheless, the growth rate in the steady-state is given by the first equation of the previous system. It is a decreasing function of the environmental tax rate.

Therefore, we obtain the conventional result of the $A K$ growth model with infinitly-lived agents: environmental policy is always harmful to growth because it reduces the rewards to capital and therefore physical capital accumulation. 


\subsection{Lifetime is finite but is not affected by pollution}

From (11), this case corresponds to $\psi=0$ : pollution have no impact on health. Therefore, the probability of death $\lambda$ does no longer depends on the tax rate $\tau: \bar{\lambda}=\frac{\delta}{\beta \theta}$. The economy is summarized by:

$$
\begin{aligned}
& g_{C, t}=\sigma[\alpha A-\Phi(\tau)-\rho]-\frac{\delta}{\beta \theta} \Delta_{\psi}(\tau) x_{t}^{-1} \\
& g_{K, t}=(1-\theta) A-x_{t}-\Phi(\tau)
\end{aligned}
$$

with $\Delta_{\psi}(\tau)=(1-\sigma) \alpha A-(1-\sigma) \Phi(\tau)+\sigma \rho+\frac{\delta}{\beta \theta} \cdot g_{C, t}$ remains an increasing function of $x_{t}$ but the influence of $\tau$ is not clear-cut because the turnover of generations (newborns are poorer than the average household) affects aggregate consumption growth and depends on $\tau$ through $\Delta_{\psi}(\tau) \cdot g_{K, t}$ is not modified.

The influence of the environmental tax on the steady-state rate of growth is then given by the sign of the following expression:

$$
\frac{1}{2}\left\{(1-\sigma) \frac{2 \bar{\lambda}+\Omega(\tau)}{\sqrt{\Omega(\tau)^{2}+4 \Delta_{\psi}(\tau) \lambda}}-(1+\sigma)\right\}
$$

with $\Omega(\tau)>0$ for all $\sigma$. In appendix A, we demonstrate that the term into brackets is negative whatever $\sigma$, therefore $\partial g^{\star} / \partial \tau<0$.

Consequently, when the lifetime of agents is finite but is not affected by pollution, the environmental policy remains harmful to growth. ${ }^{16}$ Nevertheless, as demonstrated in appendix A, by introducing an externality in aggregate consumption through the "generational turnover effect", finite lifetime modifies the size of this detrimental impact, according to the value of the intertemporal elasticity of substitution of the consumption $\sigma$. When $\sigma<1$ the negative impact of the environmental policy on the growth rate is reduced, while for $\sigma>1$ the negative impact is reinforced and the environmental policy is more detrimental. When $\sigma=1$, finite lifetime does not modify the influence of the environmental policy. The

\footnotetext{
${ }^{16}$ In a model of human capital accumulation à la Lucas (1988), the finiteness of lifetime is a sufficient condition to obtain a positive impact of the environmental policy on growth, because the turnover of generations affects the decision to educate. (proof upon request)
} 
mechanisms are similar to those of section 4 explaining the negative influence of the tax on the ratio aggregate consumption to aggregate capital $x^{\star}$ when $\sigma<1$ : they transit through the propensity to consume out of wealth $\Delta(\tau)$ and through $\Omega(\tau)$ (the aggregate consumptionphysical capital ratio at the steady-state when lifetime is infinite - see the expressions below equation 20). When $\sigma<1$, agents want to smooth consumption over time. Other things equal, a higher environmental tax will reduce the rewards to physical accumulation and therefore their non-human wealth. Consequently, agents reduce their propensity to consume out of whealth and increase their saving to compensate the reduction of their wealth. That positively influences the accumulation of physical capital and limits the effect of the decreasing interest rate: the fall in the accumulation of physical capital is reduced. This effect is reinforced by the drop of $x^{\star}$ which enables to equalize the aggregate consumption growth to the physical capital growth (Cf. section 4). At the opposite, when $\sigma>1$, agents care more about their current consumption. Consequently, they increase their propensity to consume in order to compensate the decline of their wealth and therefore they reduce their saving. This reinforces the detrimental impact of taxation on the accumulation of physical capital and therefore on growth. Furthermore $x^{\star}$ rises because aggregate consumption growth is more influenced by the increase in the interest rate than physical capital growth (Cf. section $4)$.

\subsection{Lifetime is finite and is affected by pollution}

Because the general case is very cumbersome to study, we only investigate analytically the case where $\sigma=1$. Then, $\Delta(\tau)=\mathcal{L}(\tau)+\rho$ is a decreasing function $\tau$ and $\Omega(\tau)=\bar{\Omega}$ is independent of $\tau$. Consequently, using equation (20), we have $\frac{\partial g^{\star}}{\partial \tau}>0$ if an only if

$$
\gamma \psi \frac{\mathcal{L}(\tau)[\rho+2 \mathcal{L}(\tau)]}{\Phi(\tau) \sqrt{\bar{\Omega}^{2}+4[\rho+\mathcal{L}(\tau)] \mathcal{L}(\tau)}}>1
$$

with $\Phi(\tau) \equiv[\gamma \tau]^{\frac{1}{1+\gamma}}$ and $\mathcal{L}(\tau) \equiv \frac{\delta}{\beta \theta} \Phi(\tau)^{-\psi \gamma}$. 
Because the left-hand side of this inequality is a decreasing function of $\tau,{ }^{17}$ this inequality defines a value $\hat{\tau}$ below which the condition is verified. Therefore, when pollution affects health and health influences life expectancy the relation between the environmental policy and growth is reversed U-shaped: for low values of the tax, the environmental policy promotes growth and for high values it harms growth.

To understand this result, remember that the two terms of the condition (22), when they are multiplied by $\partial \Phi(\tau) / \partial \tau$, represent the two opposite effects of the environmental $\operatorname{tax} \tau$ on the steady-state growth rate. The term in the left-hand side of the inequality $\gamma \psi \frac{\mathcal{L}(\tau)[\rho+2 \mathcal{L}(\tau)]}{\Phi(\tau) \sqrt{\bar{\Omega}^{2}+4 \mathcal{L}(\tau)[\rho+\mathcal{L}(\tau)]}}$ - captures the influence of the environmental tax on the "generational turnover effect" through life expectancy. ${ }^{18}$ The term in the left-hand side of the inequality 1 - captures the detrimental impact of the environmental taxation on the interest rate. The difference between this two effects determine the sign and the size of the environmental tax influence on growth. When the level of environmental taxation is low, the probability of death is high and the influence of the environmental taxation on the "generational turnover effect" as well. The gap is positive and important: the growth rate in the economy is positively influenced by the environmental policy. As the tax increases, the positive effect on the "generational turnover effect" reduces towards unity, because it is positively influenced by the probability of death $\mathcal{L}(\tau)$ which decreases in $\tau$. The positive effect of the tax on growth drops to zero and becomes negative for high values of $\tau$, when $\gamma \psi \frac{\mathcal{L}(\tau)[\rho+2 \mathcal{L}(\tau)]}{\sqrt{\bar{\Omega}^{2}+4 \mathcal{L}(\tau)[\rho+\mathcal{L}(\tau)]}}$ fall under unity, and the difference between the two effects becomes negative.

Because the term $\gamma \psi \frac{\mathcal{L}(\tau)[\rho+2 \mathcal{L}(\tau)]}{\Phi(\tau) \sqrt{\bar{\Omega}^{2}+4 \mathcal{L}(\tau)[\rho+\mathcal{L}(\tau)]}}$ tends towards infinity when $\tau$ approaches zero, the reversed U-shaped relation always exists when the detrimental impact of pollution on

\footnotetext{
${ }^{17}$ Its derivative with respect to $\tau$ is $\frac{\gamma \psi\left[\rho \bar{\Omega}^{2}+2 \mathcal{L}(\tau)\left(\rho^{2}+2 \bar{\Omega}^{2}+6 \rho \mathcal{L}(\tau)+4 \mathcal{L}(\tau)^{2}\right)\right]}{\Phi(\tau)\left[\bar{\Omega}^{2}+4 \mathcal{L}(\tau)(\rho+\mathcal{L}(\tau))\right]^{3 / 2}} \frac{\partial \mathcal{L}(\tau)}{\partial \tau}-\frac{\Phi(\tau)^{-2} \mathcal{L}(\tau)[\rho+2 \mathcal{L}(\tau)]}{\sqrt{\bar{\Omega}^{2}+4[\rho+\mathcal{L}(\tau)] \mathcal{L}(\tau)}} \frac{\partial \Phi(\tau)}{\partial \tau}<0$

${ }^{18}$ Remember that here, to simplify the analysis, we have fixed $\sigma=1$ and therefore the effect of $\tau$ on the propensity to consume out of wealth completely transits through the probability of death because $\Delta(\tau)$ reduces to $\rho+\mathcal{L}(\tau)$. For $\sigma \neq 1$, the influence of the environmental tax would be more complicated (see equation 20).
} 
health $\psi$ is positive. And the greater the detrimental impact of pollution on health (the higher $\psi$ ), the bigger the positive "generational turnover effect" of the environmental tax on growth, because the higher the gains in terms of life expectancy improvement. ${ }^{19}$

Using condition (22) and the definitions of $\Phi(\tau)$ and $\mathcal{L}(\tau)$, it is possible to find the impact of the other parameters on the critical value $\hat{\tau}$ (a negative sign means that the increase in the parameter diminishes the value of $\hat{\tau}$ )

\begin{tabular}{|l|c|c|c|c|c|c|c|}
\hline & $\delta$ & $\theta$ & $\beta$ & $A$ & $\rho$ & $\psi$ & $\gamma$ \\
\hline \hline$\hat{\tau}$ & + & - & - & - & + & + & $?$ \\
\hline
\end{tabular}

Table 1: Impact of the parameters on $\hat{\tau}$

When the intertemporal elasticity of substitution of consumption is unity, the environmental policy is more likely to promote growth ( $\hat{\tau}$ is higher) when public health expenditures in terms of GDP $(\theta)$ and/or the productivity in the health sector $(\beta)$ are lower and/or the influence of pollution on health $(\psi)$ is higher, and/or consumers are more impatient ( $\rho$ higher). Indeed, a higher $\beta$ or $\theta$ implies a lower probability of death $\mathcal{L}(\tau)$ for a given level of the environmental tax: the room for an improvement in life expectancy is reduced. ${ }^{20}$ Consequently the "generational turnover effect" is lower and the positive effect offsets the negative one for a smaller range of taxation level ( $\hat{\tau}$ is smaller). A higher preference for the present $\rho$ incites agents to consume more today: the propensity to consume out of wealth rises while saving and physical capital accumulation decreases. The "generational turnover effect $t^{\prime \prime}$ rises and the positive effect dominates for a greater range of taxation level ( $\hat{\tau}$ is bigger). ${ }^{21}$

\footnotetext{
${ }^{19}$ The derivative of $\gamma \psi \frac{\mathcal{L}(\tau)[\rho+2 \mathcal{L}(\tau)]}{\Phi(\tau) \sqrt{\bar{\Omega}^{2}+4 \mathcal{L}(\tau)[\rho+\mathcal{L}(\tau)]}}$ with respect to $\psi$ is positive (remembering that $\Phi(\tau)^{-\gamma}>1$ ):

$$
\frac{\gamma \psi\left[\rho \bar{\Omega}^{2}+2 \mathcal{L}(\tau)\left(\rho^{2}+2 \bar{\Omega}^{2}+6 \rho \mathcal{L}(\tau)+4 \mathcal{L}(\tau)^{2}\right)\right]}{\Phi(\tau)\left[\bar{\Omega}^{2}+4 \mathcal{L}(\tau)(\rho+\mathcal{L}(\tau))\right]^{3 / 2}} \frac{\partial \mathcal{L}(\tau)}{\partial \psi}+\gamma \frac{\Phi(\tau)^{-1} \mathcal{L}(\tau)[\rho+2 \mathcal{L}(\tau)]}{\sqrt{\bar{\Omega}^{2}+4 \mathcal{L}(\tau)[\rho+\mathcal{L}(\tau)]}}>0
$$

${ }^{20}$ Note that the room for an improvement in life expectancy is foremost determined by $\psi$, the size of the detrimental impact of pollution on health.

${ }^{21}$ The influence of $\gamma$, the elasticity of the net pollution flow $\mathcal{P}$ with respect to the ratio $K / D$, is not clear-cut
} 
To go further in the comparative statics, we use numerical simulations in the next section.

\section{NUMERICAL SIMULATIONS}

This section aims at answering two questions. First, even if we have derived analytically the influence of parameters on the value of the pollution tax $\hat{\tau}$, what are their effects on the growth rate in the economy and how do they affect the positive influence of the environmental taxation? Second, what is the influence of the intertemporal elasticity of substitution of consumption $\sigma$ on the link between the environmental policy and growth?

To answer these questions, we use numerical simulations. We first calibrate the model to obtain realistic values of the probability of death for the US economy and a realistic rate of growth. From the World Development Indicators 2005 by the World Bank, life expectancy was 77.4 years in 2003, and the public health expenditures as percentage of GDP was 6.55\%. Since the expected lifetime is the reverse of the probability of death per unit of time $\lambda$, we want $\lambda$ to be close to $1 / 77.4=0.0129$.

Table 2 summarizes the benchmark value of parameters.

\begin{tabular}{|c|c|c|c|c|c|c|c|}
\hline$\theta$ & $\alpha$ & $\delta$ & $\psi$ & $\beta$ & $\rho$ & $A$ & $\gamma$ \\
\hline \hline 0.0655 & 0.3 & 0.025 & 2 & 20 & 0.065 & 0.7 & 0.3 \\
\hline
\end{tabular}

Table 2: Benchmark value of parameters

The relation between environmental tax and the rate of growth obtained is drawn in Figure 2. The value of the environmental taxation $\hat{\tau}$ is $5.33 \%$ and the rate of growth for this value (which is also the maximum rate of growth attainable) equals $5.85 \%$. Note also that $\tau \in] 0.01,0.23$ [ to have a positive growth rate in the steady-state.

In the appendix $B$, we report the effects of a variation of the key parameters on the growth rate and on $\hat{\tau}$. Numerical simulations confirm, for example, that a higher public health expenditures in terms of GDP $(\theta)$ and a greater productivity in the health sector $(\beta)$ makes the

analytically because it affects $\Phi(\tau)$ in different ways according to its value and the value of $\tau$. Note that $\gamma$ could be set to unity without loss of generality. The qualitative results would not be modified. 
Figure 2: Benchmark case

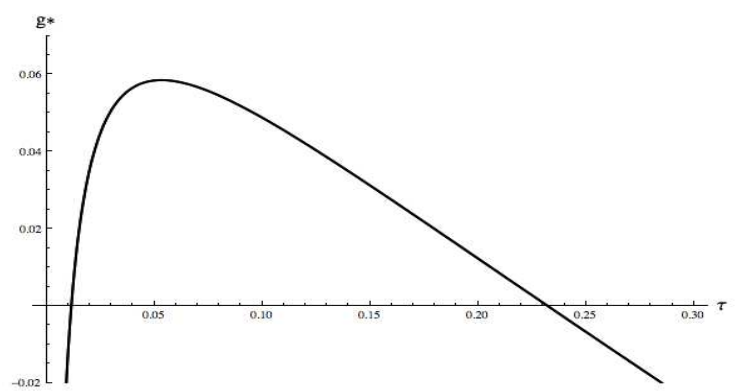

environmental policy less likely to promote growth. But the maximum rate of growth (for an environmental tax equals to $\hat{\tau}$ ) is greater than for all values of the environmental tax. As explained before, a higher $\beta$ or $\theta$ implies that the positive effect of the environmental taxation offsets the negative one for a smaller range of taxation level ( $\hat{\tau}$ is smaller) due to a smaller probability of death. Furthermore, this lower probability of death reduces the propensity to consume out of wealth. Aggregate consumption drops while saving, investment and physical capital accumulation rise and the growth rate of output is higher for each level of the environmental tax. For the same reasons, when the negative influence of pollution on health $(\psi)$ increases, the value of the environmental tax $\hat{\tau}$ rises (because the probability of death augments) and the maximum rate of growth is higher (because the augmentation of the probability of death reduces the propensity to consume out of wealth).

Consequently, even if the tax value for which the environmental tax becomes harmful to growth $(\hat{\tau})$ is lower, for each tax level higher, the growth rate is higher.

\begin{tabular}{|c|c|c|c|c|c|c|c|}
\hline$\sigma$ & 0.25 & 0.5 & 0.75 & 1 & 1.5 & 2 & 2.5 \\
\hline$\hat{\tau}$ & 0.1730 & 0.0948 & 0.0677 & 0.0533 & 0.0379 & 0.0297 & 0.0245 \\
\hline
\end{tabular}

Table 3: Impact of an increase in $\sigma$

Finally, we simulate the relation between the growth rate and the environmental tax for different values of the intertemporal elasticity of substitution $\sigma$. For the values chosen in 
Figure 3: Increase in $\sigma$ (the straightline is the benchmark case)

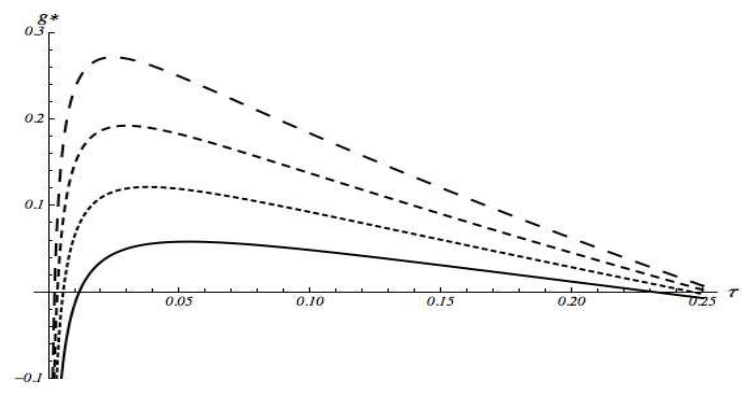

our numerical simulations, Figure 3 and Table 3 confirm our main result when $\sigma$ is different from unity: the relation between the environmental policy and growth is reversed U-shaped when pollution affects life expectancy. Furthermore, the value of the environmental taxation $\hat{\tau}$ is higher when agents want to smooth their consumption over time ( $\sigma$ is small): the environmental policy is more likely to promote growth in such a case. Indeed, besides the positive effect of the increasing lifetime itself, the increase in the propensity to consume out of wealth when $\sigma<1$ reinforces the positive effect of the environmental taxation, while when $\sigma>1$ it reduces it (as explained in section 5.2).

\section{CONCLUSION}

The aim of this article was to investigate the impact of the environmental policy on growth in an $A K$-type growth model, when finite lifetime is introduced and the link between pollution and life expectancy (through the detrimental impact of pollution on health) is taken into account.

We first demonstrate that finite lifetime introduces a "generational turnover effect" which modifies the influence of the environmental policy on growth, even if pollution does not influence life expectancy. Thus, we show that with finite lifetime independent to pollution, the detrimental impact of the environmental policy on growth is reduced when agents smooth 
their consumption over time. Indeed, by diminishing the interest rate and therefore the accumulation of physical capital and the non-human wealth of agents, the environmental taxation incites agents to reduce their propensity to consume out of wealth and to save more today. Aggregate consumption growth and investment increase, and this positive impact limits the crowding-out effect of the environmental tax.

We also demonstrate that, when the detrimental effect of pollution on health and life expectancy is taken into account, the "generational turnover effect" affects the outcome of the environmental policy through a second channel: life expectancy. In this case, the relation between environmental policy and growth is reversed U-shaped in the steady-state, whatever the value of the intertemporal elasticity of substitution of consumption. For low levels of taxation, the environmental policy promotes growth because the positive effect of a lower net flow of pollution on health and life expectancy leads the "generational turnover effect" to offset the crowding-out effect of the increasing tax. For higher levels, the environmental policy becomes harmful to growth because the room for significant health improvements is exhausted and the crowding-out effect surpasses the "generational turnover effect". Furthermore, we show that the environmental policy is more likely to promote growth when public expenditures in health is low and/or the detrimental impact of pollution on health is high (that is when the room for health improvements is higher). Finally, we make numerical simulations to investigate the influence of the intertemporal substitution rate of consumption on our result. We find that, for the value of parameters chosen, the environmental policy is more likely to promote growth when agents want to smooth consumption over time.

Consequently, the contribution of this article is twofold. First, it highlights a new mechanism through which the environmental policy affects economic growth: the generational turnover effect. Second, it demonstrates that as soon as there is a room for significant health improvements, the crowding-effect of the environmental policy may be offset and the environmental policy may have a positive impact on growth (for at least a range of environmental tax), while neither substitution between polluting and non-polluting factors nor the 
influence of the environmental quality on productivity factor exist.

The implication in terms of policy is that the efforts to reduce pollution (especially air pollution or water pollution) must be continued and reinforced with expected positive impacts on economic performances (rather than negative ones), especially in the economies where the detrimental effects of pollution on health are the most important and are predicted to highly increase and where public health expenditures in terms of GDP remain very low, like in China, Malaysia, Vietnam, India or Bangladesh (see O.E.C.D., 2008, p.259 and following). ${ }^{22}$ Even in the high-income countries of North-America and Europe where public health expenditures are high (around 7\% of the GDP), our results militate for a more stringent environmental policy with positive expected effects on growth, because O.E.C.D. (2008) projects that the negative effects of pollution on health in these countries would continue to increase in the future decades (for example, premature deaths from urban ozone exposure would treble between 2000 and 2030 (O.E.C.D., 2008, p.259)).

Finally, our results call for further investigations on the link between the environment and growth, especially by incorporating a more realistic health function in computational general equilibrium models to take into account the role played by the different actors of the health sector and their interactions, and to try to quantify the relative importance of the "generational turnover effect" highlighted in this article.

\section{REFERENCES}

Blanchard, O. (1985). Debt, deficits and finite horizon. Journal of Political Economy, 93:223247.

Bloom, D. and Canning, D. (2005). Health and economic growth: Micro and macro evidence. working papers 42 , CDDRL, Stanford IIS.

\footnotetext{
${ }^{22}$ From the World Development Indicators by the World Bank, in 2004 the public health expenditures in percentage of the GDP were $1.79 \%$ in China, $2.23 \%$ is Malaysia, $1.49 \%$ in Vietnam, $0.87 \%$ in India and Bangladesh, and $5.94 \%$ for World as a whole.
} 
Bloom, D., Canning, D., and Jamison, D. (2004). Health, wealth and welfare. Finance and Development, pages 10-15.

Brunekreef, B. and Holgate, S. T. (2002). Air pollution and health : review. The Lancet, 360:1233-1242.

Chen, S. and Liao, C. (2005). Health risk assessment on humans exposed to environmental polycyclic aromatic hydrocarbons pollution sources. Science of the Total Environment.

Currais, L. and Rivera, B. (1999). Income variation and health: Direct impact or reverse causation. Applied Economics Letters, 6:761-764.

Currais, L. and Rivera, B. (2003). The effect of health investment on growth: A causality analysis. International Advances in Economic Research, 9(4):312-324.

Ding, W., Lehrer, F., Rosenquist, J., and Audrain-McGovern, J. (2005). The impact of health on academic performance: New evidence using genetic markers. Technical report, University of Pennsylvania.

Englert, N. (2004). Fine particles and human health-a review of epidemiological studies. Toxicology Letters, 149:235-242.

Gradus, R. and Smulders, S. (1993). The trade-off between environmental care and long-term growth-pollution in three prototype growth models. Journal of Economics, 58(1):25-51.

Grossman, M. and Kaestner, R. (1997). Effects of education on health. In Behrman, J. and Stacey, N., editors, The Social Benefits of Education. University of Michigan Press.

Gutierrez, M. (2005). Dynamic inefficiency in an overlapping generation economy with pollution and health costs. Technical report, University of the Basque Country.

Heijdra, B. and Ligthart, J. (2000). The dynamic macroeconomic effects of tax policy in an overlapping generations model. Oxford Economic Papers, 52:677-701. 
Hettich, F. (1998). Growth effects of a revenue-neutral environmental tax reform. Journal of Economics, 67(3):287-316.

Howitt, P. (2005). Health, human capital and economic growth: A schumpeterian perspective. In López-casasnovas, G., Rivera, B., and Currais, L., editors, Health and economic growth, pages 19-40. MIT Press.

Kampa, M. and Castanas, E. (2007). Human health effects of air pollution. Environmental Pollution, doi:10.1016/j.envpol.2007.06.012.

Kosempel, S. (2004). Finite lifetimes and government spending in an endogenous model. Journal of Economics and Business, 56:197-210.

Kurttio, P., Pukkala, E., Kahelin, H., Auvinen, A., and Pekkanen, J. (1999). Arsenic concentrations in well water and risk of bladder and kidney cancer in Finland. Environmental Health Perspectives, 107(9):705-710.

López-Casanovas, G., Rivera, B., and Currais, L. (2005). Health and Economic Growth: Findings and Policy Implications. MIT Press.

Lucas, R. (1988). On the mechanisms of economic development. Journal of Monetary Economics, 22:3-42.

Michel, P. and Rotillon, G. (1995). Disutility of pollution and endogenous growth. Environmental and Resource Economics, 6(3):279-300.

Morris, R. (1995). Environmental health issues. Environmental Health Perspectives, 103(S8).

Nadal, M., Schuhmacher, M., and Domingo, J. (2004). Metal pollution of soils and vegetation in an area with petrochemical industry. Science of the Total Environment, 321:59-69. 
Neuberg, M., Rabczenko, D., and Moshammer, H. (2007). Extended effects of air pollution on cardiopulmonary mortality in vienna. Atmospheric Environment, doi:10.1016/j.atmosenv.2007.07.013.

O.E.C.D. (2008). OECD Environmental Outlook to 2030. Organisation for Economic Cooperation and Development, Paris.

Ono, T. (2003). Environmental tax policy and long-run economic growth. The Japanese Economic Review, 54(2):203-217.

Oueslati, W. (2002). Environmental policy in an endogenous growth model with human capital and endogenous labor supply. Economic Modelling, 19:487-507.

Paulu, C., Aschengrau, A., and Ozonoff, D. (1999). Tetrachloroethylene-contaminated drinking water in Massachusetts and the risk of colon-rectum, lung, and other cancers. Environmental Health Perspectives, 107(4):265-271.

Ricci, F. (2007). Channels of transmission of environmental policy to economic growth: A survey of the theory. Ecological Economics, 60:688-699.

Romer, P. (1986). Increasing returns and long run growth. Journal of Political Economy, 94:1002-1037.

Schuhmacher, M. and Domingo, J. (2006). Long-term study of environmental levels of dioxins and furans in the vicinity of a municipal solid waste incinerator. Environment International, 32:397-404.

Valent, F., Little, D., Bertollini, R., Nemer, L., Barbone, F., and Tamburlini, G. (2004). Burden of disease attributable to selected environmental factors and injury among children and adolescents in Europe. The Lancet, 363:2032-2039. 


\section{Appendix}

\section{A EXOGENOUS FINITE LIFETIME AND THE IMPACT OF THE ENVIRONMENTAL POLICY ON GROWTH}

The influence of the environmental on growth is given by:

$$
\left.\begin{array}{rl}
\frac{\partial g^{\star}}{\partial \tau}=\frac{\partial \Phi(\tau) / \partial \tau}{2 \Phi(\tau)}\left\{(1-\sigma) \Phi(\tau) \frac{2 \mathcal{L}(\tau)+\Omega(\tau)}{\sqrt{\Omega(\tau)^{2}+4 \Delta(\tau) \mathcal{L}(\tau)}}\right. & \\
-(1+\sigma) \Phi(\tau)+2 \gamma \psi \frac{\mathcal{L}(\tau)[\Delta(\tau)+\mathcal{L}(\tau)]}{\sqrt{\Omega(\tau)^{2}+4 \Delta(\tau) \mathcal{L}(\tau)}}
\end{array}\right\}
$$

with

$$
\begin{aligned}
& \Phi(\tau) \equiv[\gamma \tau]^{\frac{1}{1+\gamma}} \\
& \mathcal{L}(\tau) \equiv \frac{\delta}{\beta \theta} \Phi(\tau)^{-\psi \gamma} \\
& \Delta(\tau) \equiv(1-\sigma)[\alpha A-\Phi(\tau)]+\sigma \rho+\mathcal{L}(\tau)>0 \\
& \Omega(\tau) \equiv(1-\theta-\sigma \alpha) A-(1-\sigma) \Phi(\tau)+\sigma \rho>0
\end{aligned}
$$

When lifetime is infinite $\mathcal{L}(\tau)=0$ and we have

$$
\left.\frac{\partial g^{\star}}{\partial \tau}\right|_{\mathcal{L}(\tau)=0}=-\frac{\partial \Phi(\tau)}{\partial \tau}<0 .
$$

When lifetime is finite but independent from pollution, $\psi=0$ and $\mathcal{L}(\tau)=\bar{\lambda}$, and we obtain:

$$
\left.\frac{\partial g^{\star}}{\partial \tau}\right|_{\psi=0}=\frac{-\partial \Phi(\tau) / \partial \tau}{2}\left\{(1+\sigma)+(\sigma-1) \frac{2 \bar{\lambda}+\Omega(\tau)}{\sqrt{\Omega(\tau)^{2}+4 \Delta_{\psi}(\tau) \bar{\lambda}}}\right\}
$$

The influence of the environmental tax on the steady-state rate of growth is then given by the sign of the term into brackets. Because $\Omega(\tau)>0$ for all $\sigma$, we have $2 \bar{\lambda}+\Omega(\tau)>0$ and when $\sigma \geq 1$, the term into brackets is always positive: $\partial g^{\star} / \partial \tau<0$.

When $\sigma<1,0<1-\sigma<1$ and $\Delta_{\psi}(\tau)-\bar{\lambda}>0$, therefore $2 \bar{\lambda}+\Omega(\tau)<\sqrt{\Omega(\tau)^{2}+4 \Delta_{\psi}(\tau) \lambda}$ and $(1-\sigma) \frac{2 \bar{\lambda}+\Omega(\tau)}{\sqrt{\Omega(\tau)^{2}+4 \Delta_{\psi}(\tau) \bar{\lambda}}}<1+\sigma$. The term into brackets is positive, and $\partial g^{\star} / \partial \tau<0$, for all $\sigma$. 
Furthermore, the effect of the environmental policy is less detrimental on growth with finite exogenous lifetime if $\left.\left|\frac{\partial g^{\star}}{\partial \tau}\right|_{\psi=0}|<| \frac{\partial g^{\star}}{\partial \tau}\right|_{\mathcal{L}(\tau)=0} \mid$, that is

$$
1+\sigma+(\sigma-1) \frac{2 \bar{\lambda}+\Omega(\tau)}{\sqrt{\Omega(\tau)^{2}+4 \Delta_{\psi}(\tau) \bar{\lambda}}}<2
$$

Simplifying the condition becomes

$$
\sigma+(\sigma-1) \frac{2 \bar{\lambda}+\Omega(\tau)}{\sqrt{\Omega(\tau)^{2}+4 \Delta_{\psi}(\tau) \bar{\lambda}}}<1
$$

For $\sigma<1$, this condition is always verified and $\left|\frac{\partial g^{\star}}{\partial \tau}\right|_{\psi=0}|<| \frac{\partial g^{\star}}{\partial \tau}{ }_{\mathcal{L}(\tau)=0} \mid$. Conversely, when $\sigma>1$, the condition is never verified and consequently $\left.\left|\frac{\partial g^{\star}}{\partial \tau}\right|_{\psi=0}|>| \frac{\partial g^{\star}}{\partial \tau}\right|_{\mathcal{L}(\tau)=0} \mid$. When $\sigma=1,\left.\frac{\partial g^{\star}}{\partial \tau}\right|_{\psi=0}=\left.\frac{\partial g^{\star}}{\partial \tau}\right|_{\mathcal{L}(\tau)=0}$.

\section{B COMPARATIVE STATICS}

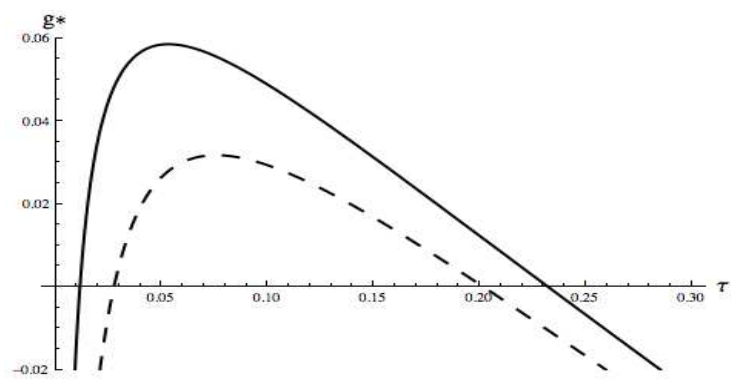

Figure 4: Increase in $\delta$ (the straightline is the benchmark case) 


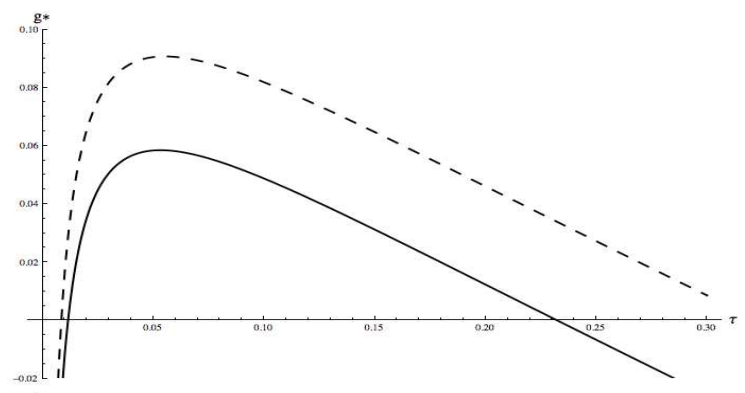

Figure 5: Increase in $\alpha$ (the straightline is the benchmark case)

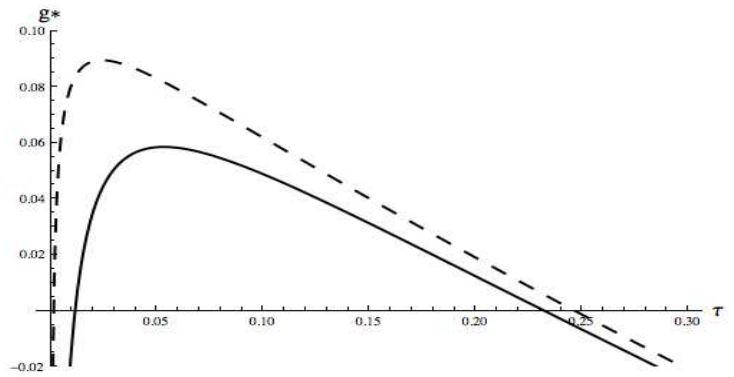

Figure 6: Decrease in $\psi$ (the straightline is the benchmark case)

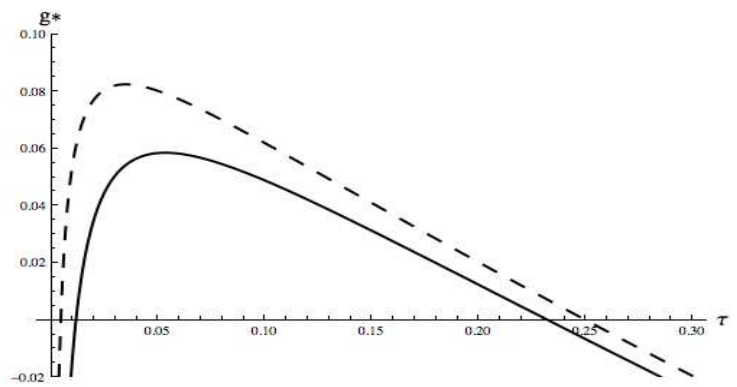

Figure 7: Increase in $\beta$ (the straightline is the benchmark case) 


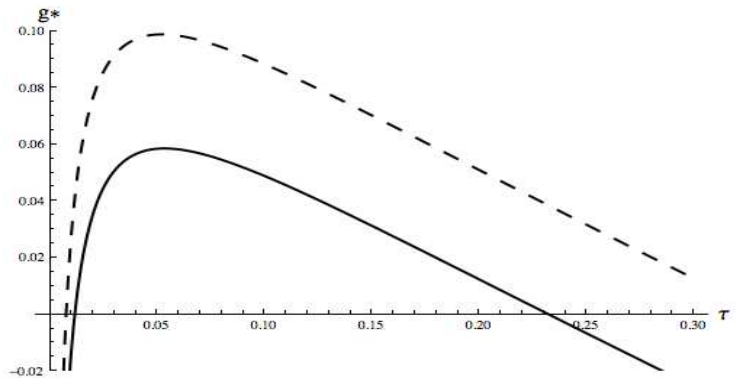

Figure 8: Decrease in $\rho$ (the straightline is the benchmark case)

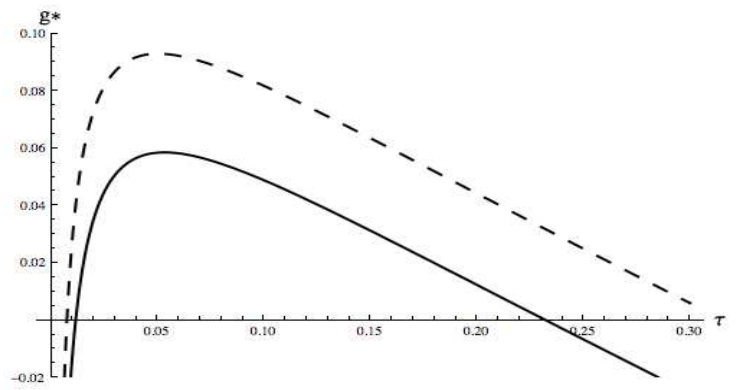

Figure 9: Increase in $A$ (the straightline is the benchmark case)

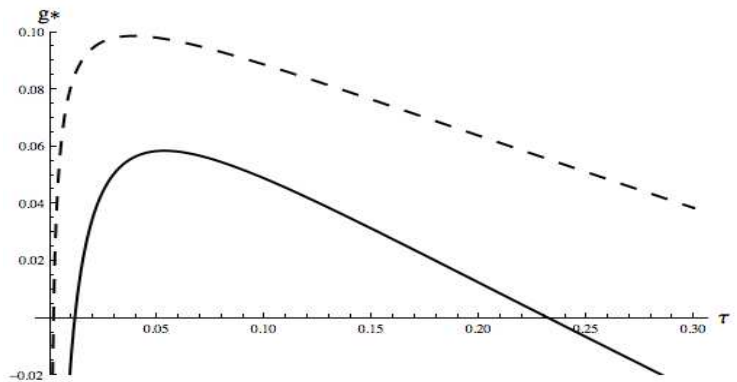

Figure 10: Decrease in $\gamma$ (the straightline is the benchmark case) 


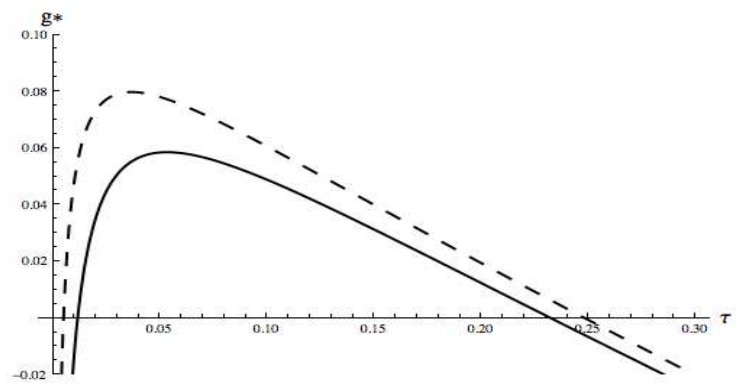

Figure 11: Increase in $\theta$ (the straightline is the benchmark case) 